\title{
Safety and Effectiveness of Coadministration of Guanfacine Extended Release and Psychostimulants in Children and Adolescents with Attention-Deficit/Hyperactivity Disorder
}

\author{
Thomas J. Spencer, M.D., Michael Greenbaum, M.D., ${ }^{2}$ \\ Lawrence D. Ginsberg, M.D., ${ }^{3}$ and William Rory Murphy, M.D. ${ }^{4}$
}

\begin{abstract}
Objective: The aim of this study was to evaluate the safety and effectiveness of guanfacine extended release (GXR) administered concomitantly with psychostimulants in children and adolescents with attention-deficit/hyperactivity disorder (ADHD) and suboptimal response to a psychostimulant alone.

Design and Methods: This was a multicenter, open-label, 9-week, dose-escalation study of 75 subjects with ADHD treated with methylphenidate (MPH) or amphetamine (AMP) alone for at least 1 month, yet with suboptimal control of ADHD symptoms. Sixty-three subjects (84.0\%) completed the study. Patients received GXR in addition to their psychostimulant. Starting with $1 \mathrm{mg}$ /day, GXR was increased weekly to the highest tolerated dose $(1,2,3$, or $4 \mathrm{mg} /$ day $)$, which was maintained through week 6. GXR was then titrated downward in 1-mg weekly decrements from week 7 through week 9. Psychostimulant treatment regimens were continued until at least week 7.

Main Outcome Measures: Safety assessments included adverse events (AEs), vital signs, physical examination, clinical laboratory tests, the Pediatric Daytime Sleepiness Scale, and the Pittsburgh Side Effects Rating Scale. Efficacy was assessed using the ADHD Rating Scale IV (ADHD-RS-IV), the Conners' Parent Rating Scale-Revised Short Form, Clinical Global Impressions, Parent Global Assessment, and Child Health Questionnaire-Parent Form.

Results: The most common treatment-related AEs were upper abdominal pain (25.3\%), fatigue (24.0\%), irritability (22.7\%), headache $(20.0 \%)$, and somnolence $(18.7 \%)$. Most AEs were mild to moderate in severity. Investigator-rated AEs due to blood pressure decreases, heart rate, or electrocardiogram findings were infrequent. Mean changes from baseline (psychostimulant monotherapy just prior to receiving GXR) to end point in ADHD-RS-IV total score were statistically significant overall: $-16.1(p<0.0001)$. Significant improvement in both subscales of the ADHD-RS-IV was observed. Improvement of symptoms was observed in a majority of subjects.

Conclusion: Coadministration of GXR and MPH or AMP was generally safe and associated with statistically significant and clinically meaningful ADHD symptom improvement in children and adolescents.
\end{abstract}

\section{Introduction}

A TTENTION-DEFICIT/HYPERACTIVITY DISORDER (ADHD) is one of the most common psychiatric disorders of childhood. An estimated $8-12 \%$ of children are affected with the disorder (Biederman and Faraone 2005). Among available treatment agents, the psychostimulants amphetamine (AMP) and methylphenidate
(MPH) are a mainstay of ADHD pharmacotherapy (Scahill et al. 2001). However, an estimated 25-30\% of patients do not respond to either MPH or AMP as monotherapy (Cantwell 1996; Scahill et al. 2001; Greenhill et al. 2002). Furthermore, a landmark 2001 National Institutes of Mental Health (NIMH) study estimated that only $56 \%$ had a complete response to optimal doses of stimulants (Swanson et al. 2001). For those individuals who do not fully

\footnotetext{
${ }^{1}$ Clinical and Research Program, Pediatric Psychopharmacology, Harvard Medical School and Massachusetts General Hospital, Boston, Massachusetts.

${ }^{2}$ Capstone Clinical Research, Libertyville, Illinois

${ }^{3}$ Red Oak Psychiatry Associates, Houston, Texas.

${ }^{4}$ Psychiatric Associates, Overland Park, Kansas.

This study was supported by Shire Development Inc.

These data were previously presented at the American Psychiatric Association (APA) Washington, D.C., May 3-6, 2008, and the Annual U.S. Psychiatric and Mental Health Congress, San Diego, California, October 30 to November 2, 2008.
} 
respond to stimulants, combination pharmacotherapy is one option that may provide more effective treatment.

Clinicians commonly prescribe a combination of medications, despite the lack of U.S. Food and Drug Administration approval and clinical research to support the use of combination therapy (Popper 1995; Brown 2004; Adler et al. 2006). Combination therapy may be prescribed to address a partial response to a medication, dose-limiting adverse events (AEs), treatment-induced or -exacerbated conditions such as sleep disturbances, or co-morbidities such as tic disorders (Adler et al. 2006). Despite its potential utility, clinicians must consider the possible risks associated with combination pharmacotherapy, such as the potential for drug-drug interactions. For this reason, careful patient monitoring is essential (Adler et al. 2006).

There is a paucity of data from controlled trials prospectively designed to assess the clinical benefits and safety of combination pharmacotherapy. Indeed, the literature is limited to case reports, anecdotal information, and a relatively small number of clinical studies, few of which were double blind or placebo controlled. Studies of combination therapy with the $\alpha_{2}$-adrenoceptor agonist clonidine and psychostimulants have generally shown benefits of combination therapy over monotherapy (Connor et al. 2000; The Tourette's Syndrome Study Group 2002; Hazell and Stuart 2003). The most recent study, a randomized, double-blind, placebocontrolled trial $(n=122)$ of children with ADHD, did not find a significant benefit of combination therapy with clonidine and MPH over MPH alone on the primary outcome measure, the Abbreviated Symptoms Questionnaire (ASQ)-Teacher Score. Additional benefits of combination therapy were seen, however, on some secondary measures (Palumbo et al. 2008). In the past, case-level data have raised safety concerns regarding the combination of MPH and clonidine (Popper 1995; Swanson et al. 1995). However, in a separate safety analysis, the authors of the study described previously concluded that they found no support for harmful interactions between clonidine and MPH in their study population (Daviss et al. 2008).

The selective $\alpha_{2 \mathrm{~A}}$-adrenoceptor agonist guanfacine has been used both in monotherapy and in combination with stimulants in the treatment of ADHD. Although clonidine and guanfacine are both $\alpha_{2}$-adrenoceptor agonists, guanfacine is more selective for the $\alpha_{2 \mathrm{~A}}$-adrenoceptor, and this selectivity has been hypothesized to account for the less sedating and hypotensive profile of guanfacine compared with clonidine (Uhlén et al. 1991; Arnsten et al. 2007).

In small pilot studies in both children and adults, monotherapy with immediate-release guanfacine has been shown to be relatively well tolerated and effective (Chappell et al. 1995; Horrigan and Barnhill 1995; Hunt et al. 1995; Dulcan et al. 1997; Scahill et al. 2001). The usefulness of immediate-release guanfacine, however, is limited clinically by its duration of action, which necessitates multiple daily doses (two to four times a day) (Chappell et al. 1995; Hunt et al. 1995; Boellner et al. 2007; Kisicki et al. 2007).

In contrast with immediate-release guanfacine, the extendedrelease formulation of guanfacine (GXR) allows effective once-daily dosing (Shojaei et al. 2006). In addition to convenience, once-daily dosing may result in a significant reduction in the peakto-trough fluctuations associated with multiple daily dosing. Maintaining therapeutic levels may also improve both the safety and efficacy profile (Kisicki et al. 2007; Swearingen et al. 2007). Of note, the bioavailability of GXR is $58 \%$ of that of immediaterelease guanfacine (Shojaei et al. 2006).
The safety and efficacy of $\operatorname{GXR}(1,2,3$, or $4 \mathrm{mg} /$ day $)$ as monotherapy for ADHD was established in two large, randomized, controlled studies $(n=345,324)$ (Biederman et al. 2008; Sallee et al. 2009). GXR was recently approved by the U.S. Food and Drug Administration for the treatment of ADHD.

Although GXR monotherapy has been studied in controlled clinical trials, combination therapy with GXR and psychostimulants has not been similarly investigated. This study evaluated the safety and effectiveness of GXR $(1,2,3$, or $4 \mathrm{mg} /$ day) coadministered with a psychostimulant (either MPH or AMP) in children and adolescents aged 6-17 years with suboptimal control of their ADHD on a psychostimulant alone.

\section{Methods}

\section{Study design and eligibility}

This open-label, dose-escalation, coadministration study was conducted at 17 centers in the United States from August to December, 2004. The study protocol was approved by Institutional Review Boards and conducted in accordance with the principles of the Declaration of Helsinki (2000) and the International Conference on Harmonisation's Guideline for Good Clinical Practice (1996).

Eligible subjects were male and female children and adolescents aged 6-17 years who satisfied the Diagnostic and Statistical Manual of Mental Disorders, 4th Edition, Text Revision (DSM-IVTR) (American Psychiatric Association 2000) criteria for a primary diagnosis of ADHD (combined, predominantly inattentive, or predominantly hyperactive/impulsive subtype) and who were functioning intellectually at an age-appropriate level. Subjects were required to have been following a stable regimen of either MPH or AMP, taking at least the minimum dose recommended by the relevant package insert for at least 1 month with suboptimal control of ADHD symptoms, as determined by the investigator. Key exclusion criteria included: Uncontrolled co-morbid psychiatric conditions, except for oppositional defiant disorder or mild anxiety; medical conditions that could confound safety assessments or prevent study completion; morbid obesity (body mass index $\geq 35$ ); hypertension or orthostatic hypotension; use of concomitant medication (other than psychostimulants) known to affect blood pressure, heart rate, or cognitive performance; and previous treatment with guanfacine. Some medications were allowed, such as antibiotics and over-the-counter medications that did not affect blood pressure, heart rate, or the central nervous system.

\section{Treatment schedule}

At baseline, all subjects were receiving a stable dose of a psychostimulant approved for the treatment of ADHD for at least 1 month with suboptimal control in the opinion of the investigator. The psychostimulant regimen was maintained at the same dose and frequency throughout the upward GXR dose-titration and dosemaintenance phases. During the upward dose-titration phase, GXR dose was started at $1 \mathrm{mg} /$ day and increased each week by $1 \mathrm{mg}$ to the highest tolerated GXR dose (1, 2, 3, or $4 \mathrm{mg} /$ day). Downward GXR dose adjustments were permitted if a given GXR dose was not tolerated. In such cases, GXR dose was reduced in 1-mg weekly decrements.

Subjects remained at the highest tolerated GXR dose through week 6. If GXR dose was adjusted for tolerability, the subject remained at the highest tolerated GXR dose for at least 3 weeks. Regardless of whether the GXR dose was adjusted, subjects con- 
tinued their stable psychostimulant regimen through the dosemaintenance phase. Subjects who terminated before completing the study were assigned to the treatment group corresponding to the last dose prior to downward titration.

GXR dose was titrated downward from the highest tolerated dose in 1-mg weekly decrements from week 7 through week 9. Until this point in the study, the dose of psychostimulant was not adjustable; however, during downward GXR dose titration, subjects and their parents were given the option of maintaining or reducing their current psychostimulant regimen. By week 10, GXR doses were discontinued, unless the subject had enrolled in a subsequent open-label extension study.

\section{Safety assessment}

The primary outcome of this study was to assess the safety of GXR coadministration with psychostimulants for the treatment of ADHD in children and adolescents with suboptimal control on a psychostimulant alone. Safety assessments included AEs, clinical laboratory tests, physical examination, vital signs, electrocardiogram (ECG) readings, the Pediatric Daytime Sleepiness Scale (PDSS), and the Pittsburgh Side Effects Rating Scale (PSERS). AEs were defined as clinically significant symptoms, conditions, or changes from the screening examination. Somnolence, sedation, and fatigue (SSF) were considered AEs of special interest: Additional analyses were performed with regard to their prevalence and intensity. Clinical chemistry (including thyroid function), hematology, and urinalysis were performed at screening, visit 6 , and visit 9. Physical examinations, including height and weight measurement, were conducted at screening and at visit 9. Vital sign measurements included seated blood pressure and pulse (taken at screening, baseline, and visits 1-10), and postural orthostatic blood pressure (at visits 4, 6, and 9). ECG readings were performed at screening, visit 6 , and visit 9 . The end point for safety assessments was defined as the last nonmissing postbaseline treatment week at week 9 (end-of-study) or earlier (early termination).

The PDSS is a self-rated assessment of daytime sleepiness that was completed at screening, baseline, and visits 1-9. The subjects responded to eight questions related to sleepiness using a Likert scale rating (never $=0$; always $=4$ ). Higher scores on the PDSS indicated greater levels of sleepiness.

The PSERS is a clinician-rated scale used to assess the severity of AEs such as dullness, tiredness, listlessness, headache, stomachache, loss of appetite, and trouble sleeping. The PSERS was completed at screening and visits $1-9$. Severity of AEs was rated as none, mild, moderate, or severe.

\section{Efficacy assessments}

The main efficacy measure was change in ADHD-RS-IV total score from baseline (when patients were receiving psychostimulants alone, just before receiving GXR) to end point, with end point defined as the last postbaseline treatment week during the upward dose-titration or dose-maintenance phases for which a valid score was obtained. The ADHD-RS-IV consists of 18 items regarding ADHD symptoms, with each item scored on a scale of 0 (no symptoms) to 3 (severe symptoms).

Additional secondary efficacy outcomes included change in score from baseline to end point for the following: the Conners' Parent Rating Scale-Revised Short Form (CPRS-R), the Clinical Global Impressions-Severity and -Improvement (CGI-S and CGI-I) scales, the Parent Global Assessment (PGA) scale, and the
Child Health Questionnaire-Parent Form (CHQ-PF50). The CPRS$\mathrm{R}$, which was used to assess duration of effect, is a parent-rated subscale of 27 items related to ADHD symptoms and problem behaviors. Items are rated on a scale of 0 (not true at all, never, seldom) to 3 (very much true, very often, very frequent). The CPRS-R was completed at 6 a.m., 6 p.m., and 8 p.m. during the baseline visit and visit 6 .

For the CGI-S, the clinician rated the severity of ADHD symptoms at baseline on a scale of 1 (no symptoms) to 7 (very severe symptoms). For the CGI-I, the clinician rated the improvement of ADHD symptoms at visit 6 on a scale of 1 (very much improved) to 7 (very much worse). The PGA, a parent-rated variation of the CGI in which the parent or caregiver evaluates the subject's symptom severity, was assessed between screening and baseline visits and again at visit 6. Responses from the CGI-I and PGA were dichotomized into two categories, "improvement" (included the responses "very much improved" and "much improved") and "no improvement" (all other responses). The CHQ-PF50 is a validated quality-of-life measure currently used in pediatric studies. In this measure, the parent answered questions that assessed 14 core health concepts, and resulted in two summary scores: One for the subject's physical well-being and another for the subject's psychosocial well-being. The transformed scores ranged from 0 to 100 , with higher scores indicative of improved health.

\section{Data analysis}

Determination of sample size by formal calculation was not applicable to this open-label study. All safety and efficacy analyses were conducted for the overall study population and by psychostimulant group. The safety population, which was used for analyses of safety, comprised all subjects who received at least one dose of study medication. Safety results were summarized using descriptive statistics and one-sample $t$-tests from baseline to postbaseline visit where applicable. The full analysis set (FAS), which was used for integrated analyses of efficacy, was defined as all subjects with a baseline and at least one postbaseline efficacy measurement.

Efficacy results were summarized using descriptive statistics and analyzed using one-sample $t$-tests to assess whether changes from baseline to end point differed significantly from zero. For the primary efficacy measure (ADHD-RS-IV), results were analyzed by age group (aged 6-12 years and 13-17 years), weight-adjusted dose group, and weight-adjusted dose group and age group. Subgroup analyses of ADHD-RS-IV inattentiveness score and ADHD-RS-IV hyperactivity/impulsivity score were also conducted by age group. Hypothesis tests were two-sided and were performed at the 5\% significance level. This study was open-label and no comparator arm was used. It was not designed or powered to evaluate a difference in safety or efficacy between psychostimulant groups or other subgroups.

\section{Results}

\section{Subject demographics}

Subject demographics and ADHD diagnoses are presented in Table 1 . Of the 75 subjects enrolled in the study, 42 received GXR and MPH $(\mathrm{GXR}+\mathrm{MPH})$ and 33 received GXR and AMP (GXR + AMP). The overall mean age of the subjects was 11.4 years, with subjects in the 6- to 12-year age group constituting $72.0 \%$ of the overall population of the study. Mean age was similar in both groups (11.1 years in the GXR + MPH group, 11.6 years in 
Table 1. Demographics and ADHD Diagnosis by Psychostimulant Group

\begin{tabular}{|c|c|c|c|}
\hline \multirow[b]{2}{*}{ Characteristics } & \multicolumn{3}{|c|}{ Psychostimulant Group } \\
\hline & Methylphenidate $(\mathrm{n}=42)$ & Amphetamine $(\mathrm{n}=33)$ & Overall $(\mathrm{n}=75$ \\
\hline \multicolumn{4}{|l|}{ Age (years) } \\
\hline Mean (SD) & $11.1(1.8)$ & $11.6(2.6)$ & $11.4(2.2)$ \\
\hline \multicolumn{4}{|l|}{ Age category (years), $n(\%)$} \\
\hline $6-12$ & $33(78.6)$ & $21(63.6)$ & $54(72.0)$ \\
\hline 13-17 & $9(21.4)$ & $12(36.4)$ & $21(28.0)$ \\
\hline \multicolumn{4}{|l|}{ Gender, $n(\%)$} \\
\hline Male & $29(69.0)$ & $26(78.8)$ & $55(73.3)$ \\
\hline Female & $13(31.0)$ & $7(21.2)$ & $20(26.7)$ \\
\hline \multicolumn{4}{|l|}{ Ethnic origin, $n(\%)$} \\
\hline White & $27(64.3)$ & $27(81.8)$ & $54(72.0)$ \\
\hline Black & $8(19.0)$ & $3(9.1)$ & $11(14.7)$ \\
\hline Hispanic & $6(14.3)$ & $2(6.1)$ & $8(10.7)$ \\
\hline Asian or Pacific Islander & 0 & $1(3.0)$ & $1(1.3)$ \\
\hline Other & $1(2.4)$ & 0 & $1(1.3)$ \\
\hline \multicolumn{4}{|l|}{ Weight (pounds) } \\
\hline Mean (SD) & $101.7(32.1)$ & $105.2(34.4)$ & $103.2(33.0)$ \\
\hline \multicolumn{4}{|l|}{ Height (inches) } \\
\hline Mean (SD) & $58.9(5.0)$ & $60.2(5.7)$ & $59.5(5.3)$ \\
\hline \multicolumn{4}{|l|}{ ADHD subtype, $n(\%)$} \\
\hline Inattentive & $7(16.7)$ & $9(27.3)$ & $16(21.3)$ \\
\hline Hyperactive/impulsive & $2(4.8)$ & $1(3.0)$ & $3(4.0)$ \\
\hline Combined & $33(78.6)$ & $23(69.7)$ & $56(74.7)$ \\
\hline \multicolumn{4}{|l|}{ Years since ADHD diagnosis } \\
\hline Mean (SD) & $3.3(2.6)$ & $4.2(3.0)$ & $3.7(2.8)$ \\
\hline Median & 3.0 & 4.0 & 4.0 \\
\hline Min, Max & 0,10 & 0,11 & 0,11 \\
\hline
\end{tabular}

Abbreviations: $\mathrm{ADHD}=$ Attention-deficit/hyperactivity disorder; $\mathrm{SD}=$ standard deviation; $\mathrm{Min}=$ minimum; $\mathrm{Max}=\operatorname{maximum}$.

the GXR + AMP group). However, age groupings differed slightly between the two psychostimulant groups: 6- to 12-year-old subjects constituted $78.6 \%$ of subjects in the GXR + MPH group, compared with $63.6 \%$ of subjects in the GXR + AMP group.

The mean weight \pm standard deviation (SD) of subjects in the study was $103.24 \pm 33.0$ pounds. For the GXR + MPH group, the mean weight $\pm \mathrm{SD}$ was $101.69 \pm 32.1$ pounds, and, for the GXR + AMP group, the mean weight \pm SD was $105.21 \pm 34.4$ ) pounds. Weight spanned a wide range in both groups: $65-180$ pounds in the GXR + MPH group and 61-197 pounds in the GXR + AMP group.

Most subjects in both psychostimulant groups were of the combined ADHD subtype (78.6\% and 69.7\% in the GXR + MPH and GXR + AMP groups, respectively). On average, subjects had been diagnosed with ADHD 3.3 years before the study in the GXR + MPH group and 4.2 years before the study in the GXR + AMP group. The time since diagnosis varied widely in both groups, ranging from 0 to 10 years in the GXR $+\mathrm{MPH}$ group and 0 to 11 years in the GXR + AMP group.

Baseline values for the main efficacy variable, ADHD-RS-IV total score, were 27.9 for the overall group, 29.1 for the GXR + MPH subgroup, and 26.3 for the GXR + AMP subgroup. CGI-S scores indicated that the subjects were moderately impaired at the start of the study $(4.0,4.2$, and 3.9 at baseline for the overall group, GXR + MPH subgroup, and GXR + AMP subgroup, respectively), despite having followed their respective psychostimulant regimens for 1 month or longer prior to baseline.

This was a dose-optimized study in which dose was titrated up to $4 \mathrm{mg} /$ day to optimal effect or to the highest tolerated dose. Mean dose of GXR at end point (the last postbaseline treatment week during the upward dose-titration or dose-maintenance phases for which a valid score was obtained) was $3.1 \mathrm{mg} /$ day. The mean weight-adjusted dose at end point was $0.07 \mathrm{mg} / \mathrm{kg}$.

\section{Safety}

Overall, 69 of 75 subjects $(92.0 \%)$ experienced a treatmentemergent adverse event (TEAE): 41 of 42 subjects (97.6\%) in the MPH group and 28 of 33 subjects $(84.8 \%)$ in the AMP group. The most common TEAEs were fatigue $(34.7 \%)$, headache $(33.3 \%)$, upper abdominal pain $(32.0 \%)$, irritability $(32.0 \%)$, somnolence (18.7\%), and insomnia (16.0\%). TEAEs judged possibly related or related to GXR that occurred in $\geq 5 \%$ of subjects are shown in Table 2. These TEAEs were experienced by a similar proportion of subjects in both psychostimulant groups (78.6\% of subjects in the GXR + MPH group and $75.8 \%$ of subjects in the GXR + AMP group). The most common possibly related or related TEAEs were upper abdominal pain $(25.3 \%)$, fatigue $(24.0 \%)$, irritability $(22.7 \%)$, headache $(20.0 \%)$, and somnolence $(18.7 \%)$. The incidences of the TEAEs were comparable between both psychostimulant subgroups except for fatigue $(28.6 \%$ in the GXR + MPH subgroup vs. $18.2 \%$ in the GXR + AMP subgroup) and irritability (14.3\% in the GXR + MPH subgroup vs. $33.3 \%$ in the GXR + AMP subgroup).

TEAEs with at least a possible relationship to GXR were mild or moderate in intensity in all subjects except for one, a 10-year-old male in the GXR + MPH group who received GXR $4 \mathrm{mg} /$ day and reported four severe TEAEs (fatigue, fecal incontinence, distorted equilibrium, and dizziness), all of which resolved without treatment and upon discontinuation of GXR. One subject experienced facial 
Table 2. Summary of TeaEs Considered Possibly Related or Related to GXR Occurring IN $\geq 5 \%$ of SubJects (SAfety Population)

\begin{tabular}{|c|c|c|c|}
\hline \multirow[b]{2}{*}{$\begin{array}{l}\text { Body system preferred } \\
\text { term }\left(\text { MedDRA }{ }^{\circledR}\right)\end{array}$} & \multicolumn{2}{|c|}{ Psychostimulant group } & \multirow[b]{2}{*}{$\begin{array}{c}\text { Overall } \\
(\mathrm{n}=75)\end{array}$} \\
\hline & $\begin{array}{l}\text { Methylphenidate } \\
\quad(\mathrm{n}=42)\end{array}$ & $\begin{array}{l}\text { Amphetamine } \\
\quad(\mathrm{n}=33)\end{array}$ & \\
\hline Total $^{\mathrm{a}}$ & $33(78.6)$ & $25(75.8)$ & $58(77.3)$ \\
\hline \multicolumn{4}{|l|}{ Gastrointestinal disorders } \\
\hline Upper abdominal pain & $10(23.8)$ & $9(27.3)$ & $19(25.3)$ \\
\hline Nausea & $3(7.1)$ & 0 & $3(4.0)$ \\
\hline \multicolumn{4}{|c|}{ General disorders and administration-site conditions } \\
\hline Fatigue & $12(28.6)$ & $6(18.2)$ & $18(24.0)$ \\
\hline \multicolumn{4}{|c|}{ Metabolic and nutrition disorders } \\
\hline Anorexia & $3(7.1)$ & $1(3.0)$ & $4(5.3)$ \\
\hline Appetite increased NOS & 0 & $2(6.1)$ & $2(2.7)$ \\
\hline \multicolumn{4}{|l|}{ Nervous system disorders } \\
\hline Headache & $8(19.0)$ & $7(21.2)$ & $15(20.0)$ \\
\hline Sedation & $4(9.5)$ & $2(6.1)$ & $6(8.0)$ \\
\hline Somnolence & $6(14.3)$ & $8(24.2)$ & $14(18.7)$ \\
\hline \multicolumn{4}{|l|}{ Psychiatric disorders } \\
\hline Anxiety & $4(9.5)$ & $3(9.1)$ & $7(9.3)$ \\
\hline Depressed mood & $1(2.4)$ & $3(9.1)$ & $4(5.3)$ \\
\hline Initial insomnia & $2(4.8)$ & $2(6.1)$ & $4(5.3)$ \\
\hline Insomnia & $6(14.3)$ & $4(12.1)$ & $10(13.3)$ \\
\hline Irritability & $6(14.3)$ & $11(33.3)$ & $17(22.7)$ \\
\hline Social avoidant behavior & $3(7.1)$ & 0 & $3(4.0)$ \\
\hline
\end{tabular}

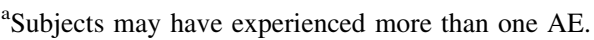

Abbreviations: TEAE $=$ Treatment-emergent adverse event; GXR $=$ guanfacine extended release; MedDRA $=$ Medical Dictionary for Regulatory Activities; NOS $=$ not otherwise specified; $\mathrm{AE}=$ adverse event.

palsy, which was felt by the investigator to be unrelated to GXR. Five of the total 75 subjects $(6.7 \%)$ discontinued due to TEAEs: 3 of the 42 subjects $(7.1 \%)$ in the GXR + MPH group and 2 of the 33 subjects $(6.1 \%)$ in the GXR + AMP group. There were no serious AEs or deaths.

When analyzed by age group, the incidence of TEAEs possibly related or related to GXR was similar between the 6- to 12-year-old subjects and 13- to 17-year-old subjects $(79.6 \%$ and $71.4 \%$, respectively). Severe TEAEs occurred in $3.7 \%$ of the 6- to 12 -yearold group, and in $0.0 \%$ of the 13- to 17-year-old group. Of the 5 discontinuations that occurred in the study, 4 were in the 6- to 12 year-old group and 1 in the 13- to 17-year-old group.

\section{SSF events}

Overall, 42 of $75(56.0 \%)$ subjects reported one or more SSF events, with similar proportions reported in the GXR $+\mathrm{MPH}$ and GXR + AMP groups (54.8\% and 57.6\%, respectively). Incidents of treatment-emergent SSF occurred in $18.7 \%$ (somnolence), $8.0 \%$ (sedation), and $34.7 \%$ (fatigue) of subjects, with no clinically meaningful differences between the psychostimulant combination subgroups in the incidences of these events. Severity of the SSF events was mild or moderate for all subjects except the previously mentioned subject who experienced severe fatigue. One subject in the GXR + AMP group discontinued due to somnolence. All sedation and somnolence events and most fatigue events resolved during the study.

The median time to onset of SSF events was 23.0 days for the GXR + MPH subgroup and 18.0 days for the GXR + AMP subgroup, coinciding with dose escalation, which was complete by visit 3 (day 21). The median duration of SSF events was 13.0 days for the GXR + MPH subgroup and 10.5 days for the GXR + AMP subgroup, with duration encompassing the time from the beginning of the first event to the end of the last event.

\section{Vital signs}

The mean on-treatment change from baseline for seated systolic and diastolic blood pressure as well as pulse are shown in Table 3. Twenty subjects exhibited decreases in blood pressure judged to be of clinical interest. Twelve of the 20 subjects were in the GXR + MPH group (2 subjects at the 3-mg/day GXR dose and 10 subjects at the 4-mg/day GXR dose); the remaining 8 subjects were in the GXR + AMP group (3 subjects at the 3-mg/day GXR dose and 5 subjects at the 4-mg/day GXR dose). Twelve subjects exhibited orthostatic blood pressure decreases. Six of the 12 subjects were in the GXR + MPH group (1 subject at the 3-mg/day GXR dose and 5 subjects at the 4-mg/day GXR dose), and the other 6 subjects were in the GXR + AMP group (2 subjects at the 3-mg/day GXR dose and 4 subjects at the 4-mg/day GXR dose). None of the subjects with blood pressure decreases reported syncope or lightheadedness.

Blood pressure decreases, although frequent, were rarely rated by investigators as AEs: 2 subjects $(2.7 \%)$ were noted by investigators to have decreased blood pressure and $1(1.3 \%)$ was noted by investigators to have unspecified hypotension.

There were no evident patterns of clinical importance with regard to hematology, clinical chemistry (including human growth hormone and cortisol), urinalysis, or physical examination results.

\section{Electrocardiogram}

Changes from screening in QT intervals using the Fridericia correction factor (QTcF) ranged from $0.01 \mathrm{msec}$ to $5.15 \mathrm{msec}$ with a mean (SD) of 3.28 (11.1) msec. Overall, no subject had an ECG 
Table 3. Mean Change in Vital Signs ${ }^{a}$ by Actual Dose ${ }^{b}$

\begin{tabular}{|c|c|c|c|c|c|}
\hline & GXR $1 \mathrm{mg} /$ day & GXR $2 \mathrm{mg} /$ day & GXR $3 \mathrm{mg} /$ day & GXR $4 \mathrm{mg} /$ day & Total \\
\hline \multicolumn{6}{|l|}{ Overall } \\
\hline SBP & $-1.3 \mathrm{mmHg}$ & $-3.9 \mathrm{mmHg}$ & $-5.8 \mathrm{mmHg}$ & $-6.9 \mathrm{mmHg}$ & $-3.5 \mathrm{mmHg}$ \\
\hline DBP & $0.8 \mathrm{mmHg}$ & $-1.7 \mathrm{mmHg}$ & $-3.0 \mathrm{mmHg}$ & $-4.1 \mathrm{mmHg}$ & $-1.4 \mathrm{mmHg}$ \\
\hline Pulse & $0.1 \mathrm{bpm}$ & $-3.9 \mathrm{bpm}$ & $-6.8 \mathrm{bpm}$ & $-9.9 \mathrm{bpm}$ & $-4.4 \mathrm{bpm}$ \\
\hline \multicolumn{6}{|l|}{ MPH } \\
\hline SBP & $-2.1 \mathrm{mmHg}$ & $-4.4 \mathrm{mmHg}$ & $-6.5 \mathrm{mmHg}$ & $-10.4 \mathrm{mmHg}$ & $-4.7 \mathrm{mmHg}$ \\
\hline DBP & $1.1 \mathrm{mmHg}$ & $-1.5 \mathrm{mmHg}$ & $-1.8 \mathrm{mmHg}$ & $-5.9 \mathrm{mmHg}$ & $-1.2 \mathrm{mmHg}$ \\
\hline Pulse & $1.6 \mathrm{bpm}$ & $-2.2 \mathrm{bpm}$ & $-4.5 \mathrm{bpm}$ & $-8.5 \mathrm{bpm}$ & $-2.8 \mathrm{bpm}$ \\
\hline \multicolumn{6}{|l|}{ AMP } \\
\hline SBP & $-0.2 \mathrm{mmHg}$ & $-3.3 \mathrm{mmHg}$ & $-5.0 \mathrm{mmHg}$ & $-1.6 \mathrm{mmHg}$ & $-2.0 \mathrm{mmHg}$ \\
\hline DBP & $0.5 \mathrm{mmHg}$ & $-2.0 \mathrm{mmHg}$ & $-4.4 \mathrm{mmHg}$ & $-1.3 \mathrm{mmHg}$ & $-1.6 \mathrm{mmHg}$ \\
\hline Pulse & $-1.8 \mathrm{bpm}$ & $-6.3 \mathrm{bpm}$ & $-9.7 \mathrm{bpm}$ & $-12.1 \mathrm{bpm}$ & $-6.4 \mathrm{bpm}$ \\
\hline
\end{tabular}

${ }^{\mathrm{a}}$ Blood pressure and pulse taken while seated.

${ }^{b}$ Values summarize all on-treatment values captured at each dose level.

Abbreviations: $\mathrm{GXR}=$ Guanfacine extended release; $\mathrm{SBP}=$ systolic blood pressure; $\mathrm{DBP}=$ diastolic blood pressure; $\mathrm{MPH}=$ methylphenidate; $\mathrm{AMP}=$ amphetamine$; \mathrm{bpm}=$ beats per minute.

finding of clinical interest or that met the ECG outlier criteria (PR interval $\geq 200 \mathrm{msec}$, QRS interval $\geq 120 \mathrm{msec}$, QT interval $\geq 480 \mathrm{msec}$, QTcF interval $\geq 500 \mathrm{msec}$, QTcB interval $\geq 500 \mathrm{msec}$, QTcF change from baseline $\geq 60 \mathrm{msec}$, QTcB change from baseline $\geq 60$ msec, or abnormal rhythm).

The proportions of subjects with a heart rate $\geq 100$ beats per minute (bpm) were comparable at screening $(10.7 \%)$ and on treatment $(11.8 \%)$. The proportion of subjects with a heart rate $\leq 50 \mathrm{bpm}$ was higher on treatment $(10.3 \%)$ than at screening (1.3\%), and was also higher in the GXR + MPH subgroup (13.2\%) than in the GXR-AMP subgroup (6.7\%). Investigator-rated AEs due to heart rate or ECG findings were infrequent; none was serious or led to discontinuation.

\section{Pediatric Daytime Sleepiness Scale}

At baseline, the mean PDSS score was 15.0. Decreases were observed at visit $6(-4.8)$ and end point $(-3.1)$.

\section{Pittsburgh Side Effects Rating Scale}

During treatment there was an increase from screening in the number of subjects reporting clinically significant dullness, tiredness, and listlessness on the PSERS. There was a decrease in the number of subjects with clinically significant loss of appetite and trouble sleeping. The psychostimulant subgroups were generally comparable and similar to the larger group.

\section{Efficacy}

Statistically significant decreases from baseline (psychostimulant only) to end point in ADHD-RS-IV total score were observed overall and in both psychostimulant combination subgroups, indicating improvement in ADHD symptoms (overall -16.1, GXR + MPH group -17.8, GXR + AMP group $-13.8 ; p<0.0001$ for all) (Fig. 1). The mean percentage reduction from baseline to end point in ADHD-RS-IV score overall was $56.0 \%$.

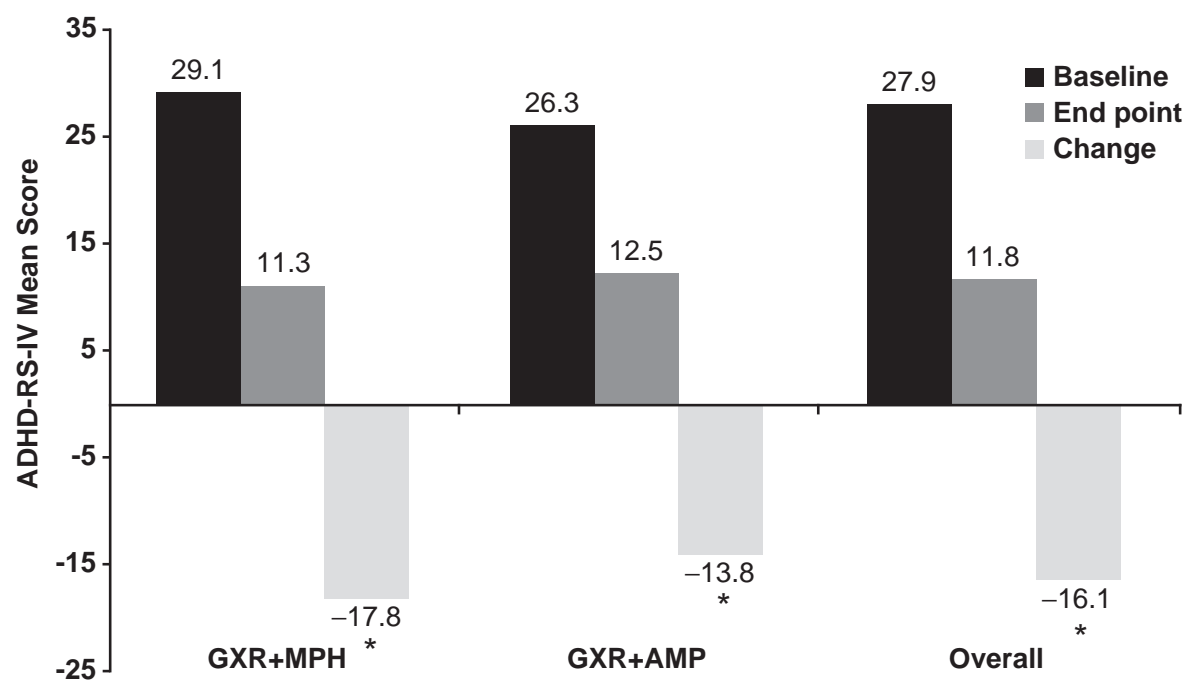

FIG. 1. ADHD-RS-IV mean scores at baseline (psychostimulant alone) and end point (psychostimulant + GXR) (ITT population). $* p<0.0001$. ADHD $=$ Attention-deficit/hyperactivity disorder; ADHD-RS-IV = ADHD Rating Scale IV; GXR = guanfacine extended release; $\mathrm{MPH}=$ methylphenidate; $\mathrm{AMP}=$ amphetamine; ITT, intent to treat. 
A subgroup analysis of the ADHD-RS-IV total score by age demonstrated statistically significant improvement for both age groups overall. For 6- to 12-year-old subjects overall, the mean improvement was $-17.3(p<0.0001)$, and for 13- to 17-year-old subjects was $-13.0(p<0.0001)$.

Statistically significant decreases from baseline to end point also were observed in the mean ADHD-RS-IV inattentiveness subscale score $(-9.2, p<0.0001)$ and in the mean ADHD-RS-IV hyperactivity/impulsivity subscale score $(-6.9, p<0.0001)$.

\section{Duration of effect}

At baseline, the mean day CPRS-R total score was 39.7 overall. Improvement was significant for the mean day CPRS-R total score $(-19.8, p<0.0001)$ as well as for all three time points $(-23.2$ at 6 p.m. [12 hours postdose], -18.5 at 8 p.m. [14 hours postdose], and -17.8 at 6 a.m. [24 hours postdose]) (all $p<0.0001$ ) (Fig. 2).

\section{Clinical Global Impressions and Parent Global Assessment}

The baseline CGI-S score was 4.0, indicating moderate impairment at baseline despite treatment with a psychostimulant at the minimum recommended dose or higher for 1 month or longer. The percentage of subjects showing improvement at end point on the dichotomized CGI was $73.0 \%$, with $27.0 \%$ showing no improvement. On the dichotomized PGA, $84.1 \%$ of subjects showed improvement, with $15.9 \%$ of subjects showing no improvement.

\section{Child Health Questionnaire-Parent Form}

The mean CHQ-PF50 baseline physical summary score was 56.2, and the mean psychosocial score was 37.3, suggesting that the every day quality of life for the subjects' families was nega- tively impacted by the subjects' ADHD. No statistically significant improvement occurred at end point in the CHQ-PF50 physical summary score. Mean improvement for the CHQ-PF50 psychosocial score was 10.2 , which was statistically significant $(p<0.0001)$.

\section{Discussion}

The use of combination pharmacotherapy necessitates establishing the safety and efficacy of the proposed therapeutic regimen. Presently, there is little evidence regarding the safety of combination pharmacotherapy in the treatment of ADHD. Although previous case-level data have raised concerns about some combinations of pharmacotherapies for ADHD, there is a lack of systematically collected data (Popper 1995; Swanson et al. 1995).

Previous studies of GXR have supported its safety and efficacy as monotherapy in the treatment of ADHD (Biederman et al. 2008; Sallee et al. 2009). This study was designed to assess the safety and effectiveness of GXR combined with either MPH or AMP in children and adolescents aged 6-17 years with ADHD who had a suboptimal response to psychostimulant alone.

This study found that GXR dosed to maximum tolerability (up to $4 \mathrm{mg} /$ day) was generally safe and effective when used in combination with MPH or AMP for the treatment of ADHD. Although $77.3 \%$ of subjects experienced a TEAE judged to be related to GXR, most of these events were mild or moderate and did not lead to discontinuation. Similarly, although $56.0 \%$ of subjects experienced SSF events, most of these events were mild or moderate and did not lead to discontinuation.

Coadministration of GXR with either MPH or AMP did not produce a unique pattern of AEs apart from what has been observed during monotherapy with either psychostimulant or GXR alone. Of the most common TEAEs reported in this study, irritability, headache, abdominal pain, and insomnia are known side effects of

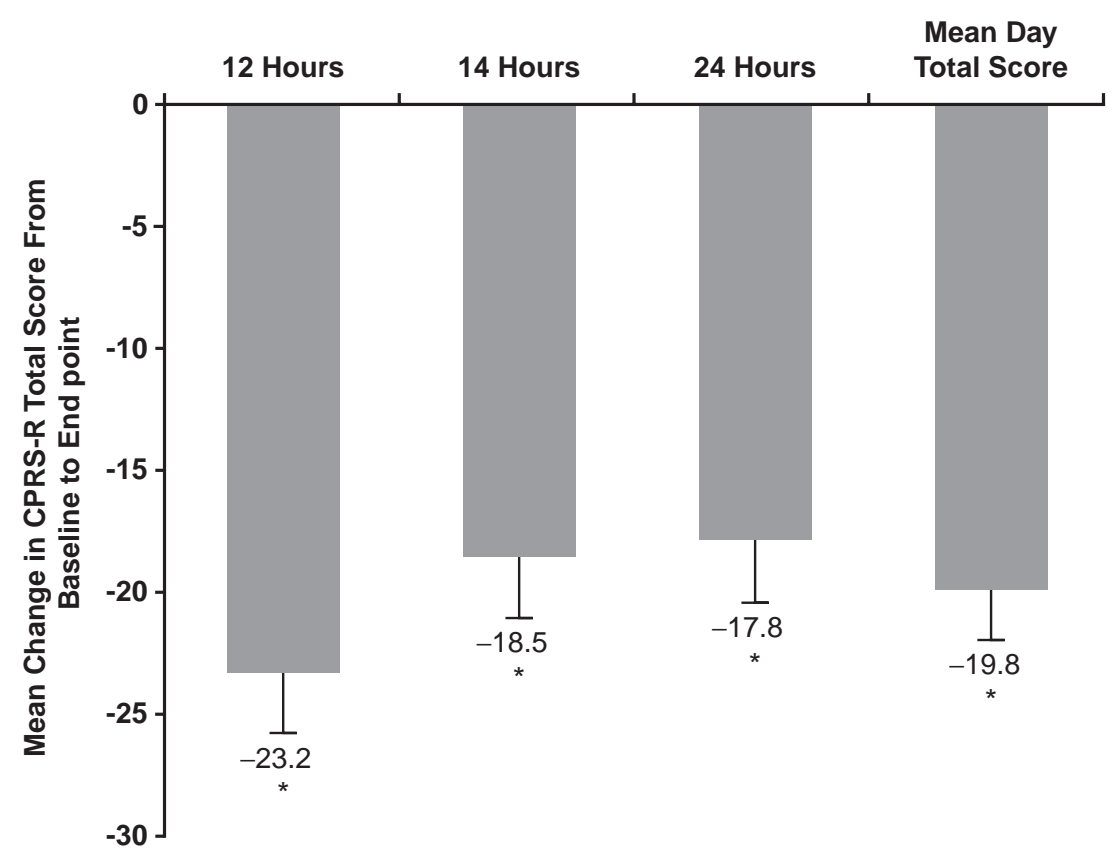

FIG. 2. Mean change in CPRS-R total score from baseline (psychostimulant alone) to end point (psychostimulant + GXR) (FAS). End point is the last postbaseline treatment week of the dose-titration and dose-maintenance phases (i.e., weeks 1-6 or days 1-42) for which a valid assessment was obtained. Mean day total scores were determined by averaging the total scores obtained at the three administrations. $* p<0.0001$. CPRS-R $=$ Conners' Parent Rating Scale-Revised Short Form; GXR = guanfacine extended release; FAS $=$ full analysis set. 
psychostimulant pharmacotherapy, whereas fatigue and somnolence have been reported as AEs in previous clinical trials of GXR (Adderall XR [package insert]; Biederman et al. 2008; Concerta [package insert]; Sallee et al. 2009). Furthermore, coadministration of GXR and either psychostimulant did not increase sleepiness, as noted by the decrease in PDSS scores assessed at visit 6 and at the end of the study.

In this study, higher doses of GXR were associated with greater mean decreases in systolic blood pressure, diastolic blood pressure, and pulse, although this relationship was not seen in the GXR + AMP group at the highest actual dose of GXR analyzed. Although no syncopal events were observed in this trial, clinicians should monitor blood pressure closely when higher doses of GXR are used.

In this patient population of children and adolescents with suboptimal control of ADHD symptoms on psychostimulants alone, there was statistically significant and clinically meaningful improvement in ADHD symptoms when GXR was given in addition to the psychostimulant. The significant decreases from baseline to end point in mean ADHD-RS-IV Inattentiveness and Hyperactivity/Impulsivity subscale scores observed in this study indicate specific benefits of GXR in the treatment of ADHD symptoms. This is consistent with data from earlier studies showing that the beneficial effects of guanfacine in the treatment of ADHD are dissociable from any sedative or hypotensive actions (Scahill et al. 2001). Although this was an open-label study, the mean decrease in ADHD-RS-IV scores was sufficient to bring the children to the normal range, a finding that deserves further exploration with a placebo-controlled study.

\section{Limitations}

Several issues should be considered regarding the results of this study. The exclusion criteria used in the study may have been selected for a study population that did not reflect the real-world population of children and adolescents with ADHD. For example, the subjects in the study were suboptimal responders to psychostimulant pharmacotherapy, possibly resulting in greater tolerability for the combination pharmacotherapy than if psychostimulantnaïve subjects had been included. Individuals with co-morbid disorders other than oppositional defiant disorder and mild anxiety-such as major depression and bipolar disorder-were ineligible for study participation, although they may represent a large portion of the population of individuals with ADHD (Milberger et al. 1995; Scahill and Schwab-Stone 2000).

Interpretation of subgroup analyses is limited by small sample sizes in this study as well as the lack of a comparator arm. The limited number of subjects did not allow detection of rare AEs and differences in effect between sexes, ages, and ethnic backgrounds in this diverse study population. The short duration of the study precludes the availability of information on long-term treatment. Due to the nature of this nonrandomized study, we could not address whether differences between the two psychostimulant groups were significant. The GXR + MPH subgroup had a higher mean baseline ADHD-RS-IV score, a higher proportion of 6 to 12 year olds, and a lower mean baseline weight, compared with the GXR + AMP subgroup. These differences may explain the differences in effectiveness results between the psychostimulant subgroups.

Because the primary goal of this trial was to examine the safety of coadministration of GXR and psychostimulants in children and adolescents with ADHD, there are many limitations to interpretation of the effectiveness outcomes. The open-label design of the study also did not allow for firm conclusions about efficacy, although it may have suggested possible efficacy. Furthermore, the design of this study does not provide data on whether combination therapy was required to achieve efficacy in these subjects. Whether a patient had suboptimal control of ADHD symptoms, and was therefore eligible for participation in the study, was determined by the judgment of the investigator. Although the assessment of suboptimal control was made through clinical judgment and corroborated by baseline assessments of symptom severity on several subscales, no data were available regarding the length of time that patients were on their respective psychostimulant medication, and the extent of dose titration for their psychostimulant. Therefore, it cannot be determined from these data whether additional benefits could have been gained by more optimal psychostimulant dosing or whether the improvements observed were due to an additive effect of GXR and psychostimulant coadministration. Conversely, because psychostimulant medication was not discontinued during this study, it is also unknown whether similar efficacy would have been maintained with GXR treatment alone.

Nevertheless, the magnitude of the decrease in ADHD-RS-IV, a validated and commonly used outcome measure, placed the children within normal range for that measure of effectiveness in ADHD studies, a finding that deserves further exploration with a placebo-controlled study. The results from this study are in line with findings reported by other investigators (Brown 2004; Adler et al. 2006) in terms of the safety and efficacy of combination pharmacotherapy involving coadministration of nonstimulants and stimulants. The results lend support to the use of GXR in combination pharmacotherapy with psychostimulants for the treatment of ADHD. Additional placebo-controlled studies are needed to make these findings more generalizable to the general population of individuals with ADHD and to further explore the efficacy of this treatment regimen.

\section{Conclusions}

To date there have been little data supporting the safety and efficacy of combination therapy for ADHD. Although more research is needed, the results of the present study support the safety and effectiveness of coadministration of GXR and AMP or MPH for the treatment of ADHD in patients with suboptimal responses to stimulants. The most common types of AEs observed in this study with combination therapy have been reported in previous studies of monotherapy with either stimulants or GXR. Furthermore, although the design of this study cannot directly address the question of whether coadministration of GXR with a stimulant augments the effects of the stimulant, the results indicate a reduction in ADHD symptoms with GXR treatment beyond that seen with AMP or MPH alone. These reductions brought mean ADHD-RS-IV scores in subjects who had not responded to stimulants into the normal range, a finding that should be explored further.

\section{Disclosures}

Dr. Thomas Spencer receives research support from, is a speaker for, or is on the advisory board of the following sources: Shire Laboratories Inc., Eli Lilly and Company, GlaxoSmithKline, Janssen Pharmaceutical, McNeil Pharmaceutical, Novartis AG, Cephalon, Inc., Pfizer Inc, and the National Institute of Mental Health. Dr. Michael Greenbaum receives research support from, or is a speaker for: Abbott Laboratories, AstraZeneca, Eli Lilly and Company, Johnson and Johnson, Novartis, Pfizer Inc, OrthoMcNeil, Shire Pharmaceutical Inc., and Takeda. Dr. Lawrence 
Ginsberg receives research support from, is a speaker for, or is consultant for the following sources: Abbott Laboratories, Alkermes, AstraZeneca, Bristol-Myers Squibb, Cephalon, Cyberonics, Forest, GlaxoSmithKline, Janssen, Jazz, JDS Pharmaceuticals, Eli Lilly and Company, McNeil, Neurocrine Biosciences, New River, Novartis, Noven Pharmaceuticals, Organon, OrthoMcNeil, Otsuka, Pamlab, Pfizer Inc, Sanofi-Aventis, Schwarz, Sepracor, Shire Pharmaceutical Inc., Takeda, UCB Pharma, Validus, and Wyeth. Dr. William Murphy received research support from Shire Pharmaceutical Inc.

\section{Acknowledgment}

Editorial assistance was provided by Jennifer Steeber, Ph.D., of Health Learning Systems, part of CommonHealth, Parsippany, New Jersey.

\section{References}

Adderal XR (package insert). Wayne (Pennsylvania): Shire US Inc., 2009.

Adler LA, Reingold LS, Morrill MS, Wilens TE: Combination pharmacotherapy for adult ADHD. Curr Psychiatry Rep 8:409-415, 2006.

American Psychiatric Association. Diagnostic and Statistical Manual of Mental Disorders, $4^{\text {th }}$ edition, Text Revision (DSM-IV-TR). Washington (DC): American Psychiatric Association.

Arnsten AF, Scahill L, Findling RL: Alpha-2 adrenergic receptor agonists for the treatment of attention-deficit/hyperactivity disorder: emerging concepts from new data. J Child Adolesc Psychopharmacol 17:393-406, 2007.

Biederman J and Faraone SV: Attention-deficit hyperactivity disorder. Lancet 366:237-248, 2005.

Biederman J, Melmed RD, Patel A, McBurnett R, Konow J, Lyne A, Scherer N, for the SPD503 Study Group: A randomized, doubleblind, placebo-controlled study of guanfacine extended release in children and adolescents with attention-deficit/hyperactivity disorder. Pediatrics 121:e73-e84, 2008.

Boellner SW, Pennick M, Fiske K, Lyne A, Shojaei A: Pharmacokinetics of a guanfacine extended-release formulation in children and adolescents with attention-deficit-hyperactivity disorder. Pharmacotherapy 27:1253-1262, 2007.

Brown TE: Atomoxetine and stimulants in combination for treatment of attention deficit hyperactivity disorder: Four case reports. J Child Adolesc Psychopharmacol 14:129-136, 2004.

Cantwell DP: Attention deficit disorder: A review of the past 10 years. J Am Acad Child Adolesc Psychiatry 35:978-987, 1996.

Chappell PB, Riddle MA, Scahill L, Lynch KA, Schultz R, Arnsten A, Leckman JF, Cohen DJ: Guanfacine treatment of comorbid attention-deficit hyperactivity disorder and Tourette's syndrome: Preliminary clinical experience. J Am Acad Child Adolesc Psychiatry 34:1140-1146, 1995.

Concerta (package insert). Titusville (New Jersey): McNeil Pediatrics, 2009.

Connor DF, Barkley RA, Davis HT: A pilot study of methylphenidate, clonidine, or the combination in ADHD comorbid with aggressive oppositional defiant or conduct disorder. Clin Pediatr 39:15-25, 2000.

Daviss WB, Patel NC, Robb AS, McDermott MP, Bukstein OG, Pelham WE Jr, Palumbo D, Harris P, Sallee FR: Clonidine for attention-deficit/hyperactivity disorder: II. ECG changes and adverse events analysis. J Am Acad Child Adolesc Psychiatry 39:15$25,2008$.
Dulcan M and the Work Group on Quality Issues: Practice parameters for the assessment and treatment of children, adolescents, and adults with attention-deficit/hyperactivity disorder. J Am Acad Child Adolesc Psychiatry 36(Suppl 10):85S-121S, 1997.

Greenhill LL, Pliszka S, Dulcan MK, Bernet W, Arnold V, Beitchman J, Benson RS, Bukstein O, Kinlan J, McClellan J, Rue D, Shaw JA, Stock S: Practice parameter for the use of stimulant medications in the treatment of children, adolescents, and adults. J Am Acad Child Adolesc Psychiatry 41(Suppl 2):26S-49S, 2002.

Hazell PL and Stuart JE: A randomized controlled trial of clonidine added to psychostimulant medication for hyperactive and aggressive children. J Am Acad Child Adolesc Psychiatry 42:886-894, 2003.

Horrigan JP and Barnhill LJ: Guanfacine for treatment of attentiondeficit hyperactivity disorder in boys. J Child Adolesc Psychopharmacol 5:215-223, 1995.

Hunt RD, Arnsten AFT, Asbell MD: An open trial of guanfacine in the treatment of attention-deficit hyperactivity disorder. J Am Acad Child Adolesc Psychiatry 34:50-54, 1995.

Kisicki JC, Fiske K, Lyne A: Phase I, double-blind, randomized, placebo-controlled, dose-escalation study of the effects on blood pressure of abrupt cessation versus taper down of guanfacine extended-release tablets in adults aged 19 to 24 years. Clin Ther 29:1967-1979, 2007.

Milberger S, Biederman J, Faraone SV, Murphy J, Tsuang MT: Attention deficit hyperactivity disorder and comorbid disorders: Issues of overlapping symptoms. Am J Psychiatry 152:1793-1799, 1995.

Palumbo DR, Sallee FR, Pelham WE Jr, Bukstein OG, Daviss WB, McDermott MP: Clonidine for attention-deficit/hyperactivity disorder: I. Efficacy and tolerability outcomes. J Am Acad Child Adolesc Psychiatry 47:180-188, 2008.

Popper CW: Combining methylphenidate and clonidine: Pharmacologic questions and news reports about sudden death. J Child Adolesc Psychopharmacol 5:157-166, 1995.

Sallee F, McGough J, Wigal T, Donahue J, Lyne A, Biederman J, for the SPD503 Study Group: Guanfacine extended release in children and adolescents with attention-deficit/hyperactivity disorder: A placebo-controlled trial. J Am Acad Child Adolesc Psychiatry 48:155-165, 2009.

Scahill L and Schwab-Stone M: Epidemiology of ADHD in schoolage children. Child Adolesc Psychiatr Clin N Am 9:541-555, vii, 2000.

Scahill L, Chappell PB, Kim YS, Schultz RT, Katsovich L, Shepherd E, Arnsten AF, Cohen DJ, Leckman JF: A placebo-controlled study of guanfacine in the treatment of children with tic disorders and attention deficit hyperactivity disorder. Am J Psychiatry 158:10671074, 2001.

Shojaei AH, Chang R-K, Pennick M: Guanfacine extended release tablets as treatment for attention-deficit/hyperactivity disorder: formulation characteristics. Poster presented at the U.S. Psychiatric \& Mental Health Congress, November 16-19, 2006, New Orleans, Louisiana.

Swanson JM, Flockhart D, Udrea D, Cantwell DP, Connor D, Williams L: Clonidine in the treatment of ADHD: Questions about safety and efficacy. J Child Adolesc Psychopharmacol 5:301-304, 1995.

Swanson JM, Kraemer HC, Hinshaw SP, Arnold LE, Conners CK, Abikoff HB, Clevenger W, Davies M, Elliott GR, Greenhill LL, Hechtman L, Hoza B, Jensen PS, March JS, Newcorn JH, Owens EB, Pelham WE, Schiller E, Severe JB, Simpson S, Vitiello B, Wells K, Wigal T, Wu M: Clinical relevance of the primary findings of the MTA: Success rates based on severity of ADHD and ODD symptoms at the end of treatment. J Am Acad Child Adolesc Psychiatry 40:168-179, 2001. 
Swearingen D, Pennick M, Shojaei A, Lyne A, Fiske K: A phase I, randomized, open-label, crossover study of the single-dose pharmacokinetic properties of guanfacine extended-release 1-, 2-, and 4-mg tablets in healthy adults. Clin Ther 29:617-625, 2007.

The Tourette's Syndrome Study Group: Treatment of ADHD in children with tics: A randomized controlled trial. Neurology 58:527-536, 2002.

Uhlén $S$ and Wikberg JES: Delineation of rat kidney $\alpha_{2 \mathrm{~A}^{-}}$and $\alpha_{2 \mathrm{~B}^{-}}$ adrenoceptors with $\left[{ }^{3} \mathrm{H}\right] \mathrm{RX} 821002$ radioligand binding: Computer modelling reveals that guanfacine is an $\mathrm{a}_{2 \mathrm{~A}}$-selective compound. Eur J Pharmacol 202:235-243, 1991.
Address correspondence to:

Thomas J. Spencer, M.D.

Associate Chief, Clinical and Research Program Pediatric Psychopharmacology Massachusetts General Hospital Associate Professor of Psychiatry Harvard Medical School 55 Fruit Street, WAW6A Boston, MA 02114

E-mail: tspencer@partners.org 